\title{
Protée
}

\section{Elmyna Bouchard. Illuminer le grain}

\section{Annie Lafleur}

Volume 36, numéro 2, automne 2008

Éthique et sémiotique du sujet

URI : https://id.erudit.org/iderudit/019019ar

DOI : https://doi.org/10.7202/019019ar

Aller au sommaire du numéro

Éditeur(s)

Département des arts et lettres - Université du Québec à Chicoutimi

ISSN

0300-3523 (imprimé)

1708-2307 (numérique)

Découvrir la revue

Citer cet article

Lafleur, A. (2008). Elmyna Bouchard. Illuminer le grain. Protée, 36(2), 50-58. https://doi.org/10.7202/019019ar d'utilisation que vous pouvez consulter en ligne.

https://apropos.erudit.org/fr/usagers/politique-dutilisation/ 


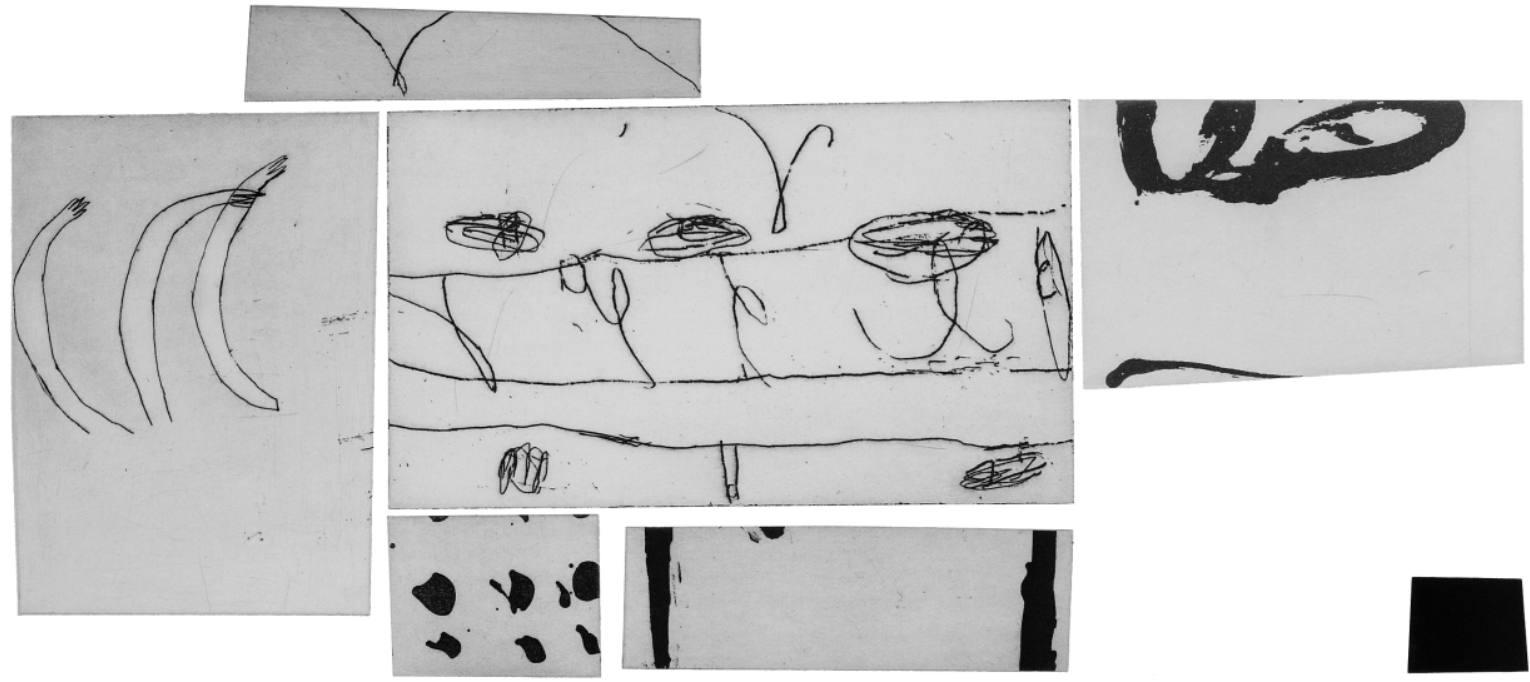

Cahier de solfège page 1, 2006. Eau-forte et relief, $56 \times 76 \mathrm{~cm}$. 


\section{EMYNA BOUCHARD}

\section{IUUMINER LE GRAIN}

Une présentation d'Annie Lafleur

Le dessin, écrit Merleau-Ponty, nous concernera comme une parole décisive, il réveillera en nous le profond arrangement qui nous a installés dans notre corps et par lui dans le monde, il portera le sceau de notre finitude, mais ainsi, et par là même, il nous conduira à la substance secrète de l'objet dont tout à l'heure nous n'avions que l'enveloppe.

(1969: 208-209; nous soulignons)

Emyna Bouchard emprunte la trajectoire clandestine des bouts de papier soigneusement encrés, étudiés, pliés, tandis que la table ferme l'ensemble aux quatre coins, qui débordent par la force des fragments étalés. Le trait ne stope jamais sa fuite spatiale, car il la conçoit sans arrêt dans l'action d'un étirement, d'une vrille, d'un jaillissement. Mais cette parole décisive qui prend de l'expansion le fait par murmures, soupirs, petits rires. Bien vite, ces œuvres reconduisent la substance secrète de l'objet à la frontière de nos sens, sous l'emprise $d^{\prime}$ 'une tonalité qui évoque une odeur ou d'un tracé qui pique notre mémoire d'enfant. Elles nous ramènent par tendres extraits, là où l'œil ne s'était pas posé depuis longtemps.

Le travail de l'artiste est constitué d'horizons purs dans lesquels notre perception devient un nombre transfini. Quelque chose d'inédit se pose incidemment avec autant de mémoire que d'étrangeté. Comme si, là, le soleil se levait selon sa propre équation algébrique. Cette science gravée propage avec assurance l'intense plaisir du tracé. II transperce et propulse sa couleur comme un petit cavalier sur sa monture à travers tous les paysages possibles, pénètre le papier, rougit, respire et meurt en de forts éclats d'encre noire, finement perlés. Il éclate en jaune couronné ou sanglote tranquillement en fines rayures, dans la pulpe même du papier.

L'œuvre d'Elmyna Bouchard est Stiftung, de sorte qu'elle «désigne [...] cette fécondité indéfinie de chaque moment du temps, qui justement parce qu'il est singulier et qu'il passe, ne pourra jamais cesser d'avoir été ou d'être universellement» (ibid.: 96). En cela, les papiers agissent comme l'oscillographe qui note les variations d'une grandeur physique en fonction du temps: «Le but est de marquer sur le papier une trace de notre 
contact avec [l']objet et [l]e spectacle, en tant qu'ils font vibrer notre regard, virtuellement notre toucher, nos oreilles, notre sentiment du hasard ou du destin ou de la liberté » (ibid.: 208). Ensembles, spectacle et objet inconnus, ils forment une vie de papier, un cosmos qui fait porte ouverte le temps d'un dessin. Si le tracé des collages s'achève brusquement, il réapparaît toutefois avec bonheur par cette mathématique imaginaire qui régit toute composition. Les règles planimétriques rêvent et se dispersent dans l'arabesque, «vers une signification qui n'était nulle part avant elle» (ibid.: 211).

Les vivres chromatiques sont renfloués au contact du support en de minuscules spectacles nucléiques, savante cadence aux pastilles colorées, galaxie sous-marine rallumée sur la plage en faisceaux paraboliques. La couleur parade comme un gros bonbon englouti, un peu fondu sur le pouce. L'œuvre s'abandonne à une conquête d'illumination du grain, comme y parvient la neige ou le pollen sur fond d'atmosphère. Tout devient visible. Le papier retrouve une façon d'être sans synonyme.

L'artiste exprime et imprime avec grâce ce qui se cache au fond d'un pigment, sur la trame humide de I'encre, dans l'intime repli du papier, en une « résonance secrète par laquelle notre finitude s'ouvre à l'être du monde et se fait poésie» (ibid.: 209). Elle restitue les figurants au premier plan: la fibre, le grain, le pli, la tonalité doivent jouer leur vie, dans tous les costumes. Le rideau de théâtre est ensuite découpé et mis en scène comme autant de personnages en quête $d^{\prime}$ une petite pièce pour papier.

Merleau-Ponty, M. [1969] : La Prose du monde, Paris, Gallimard.
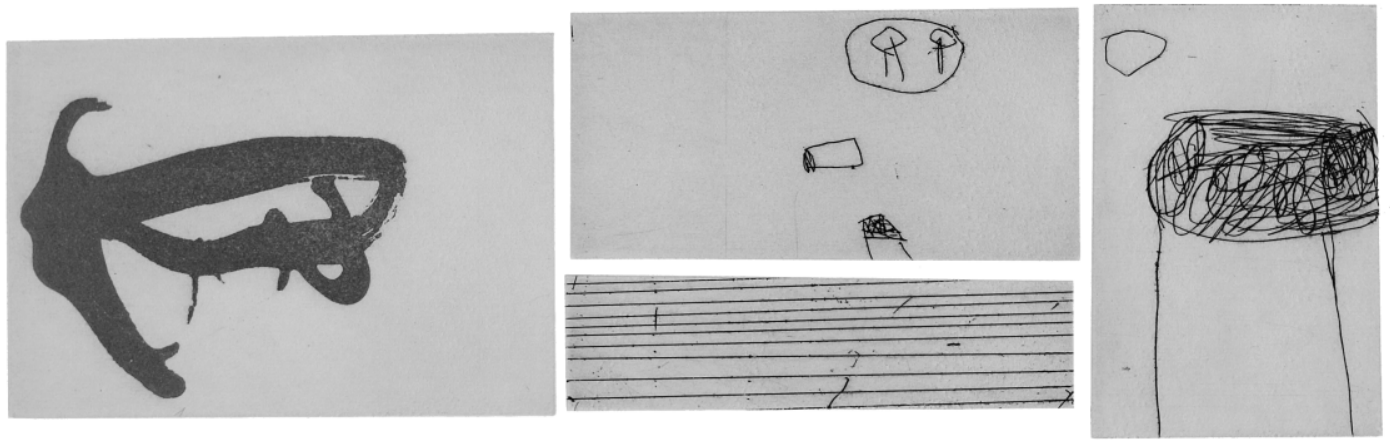

Lipstick, 2006. Eau-forte, $33 \times 57 \mathrm{~cm}$. 


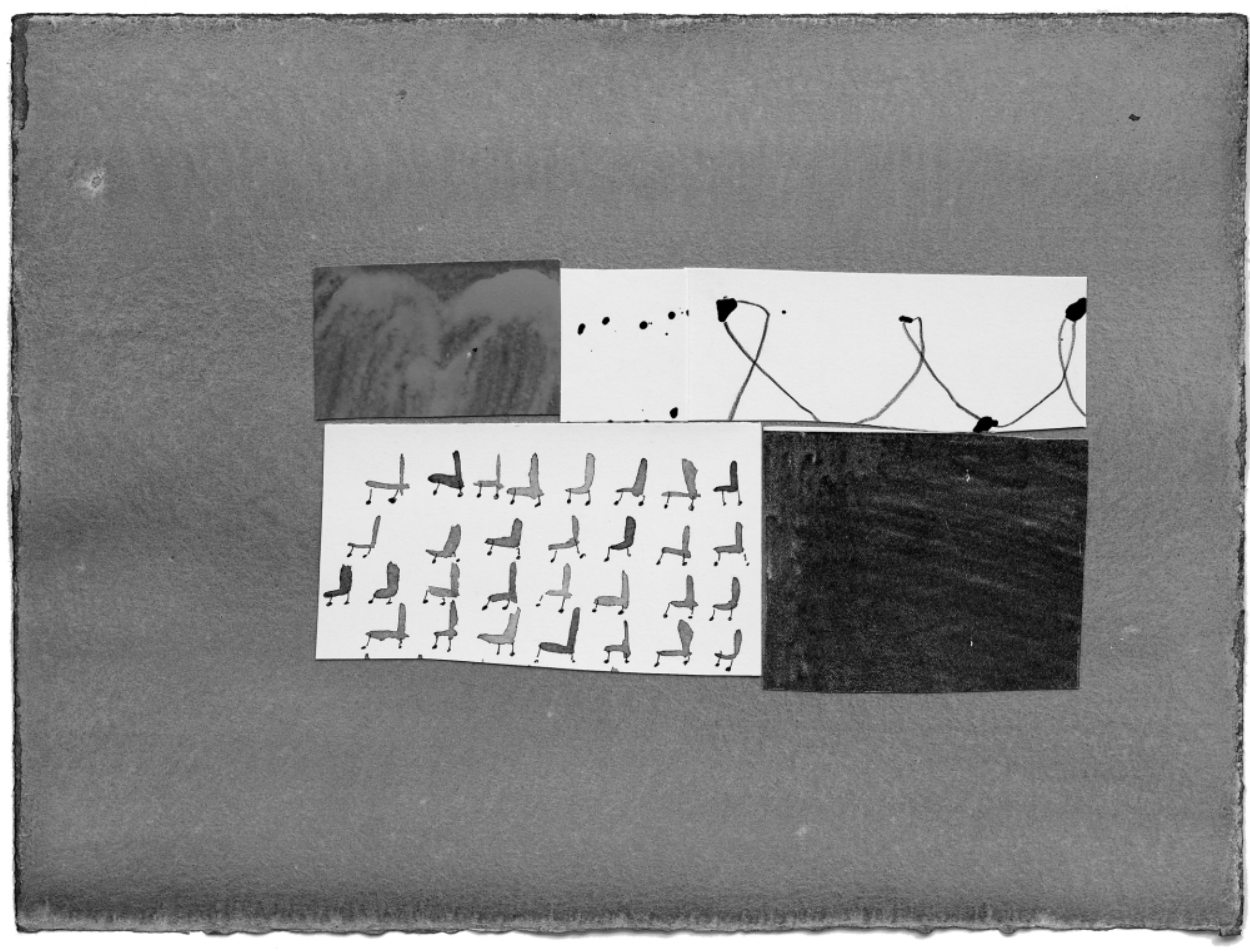

Petite pièce ouverte $n^{\circ} 36,2007$. Encre, acrylique et collage sur papier, $56 \times 76 \mathrm{~cm}$. 


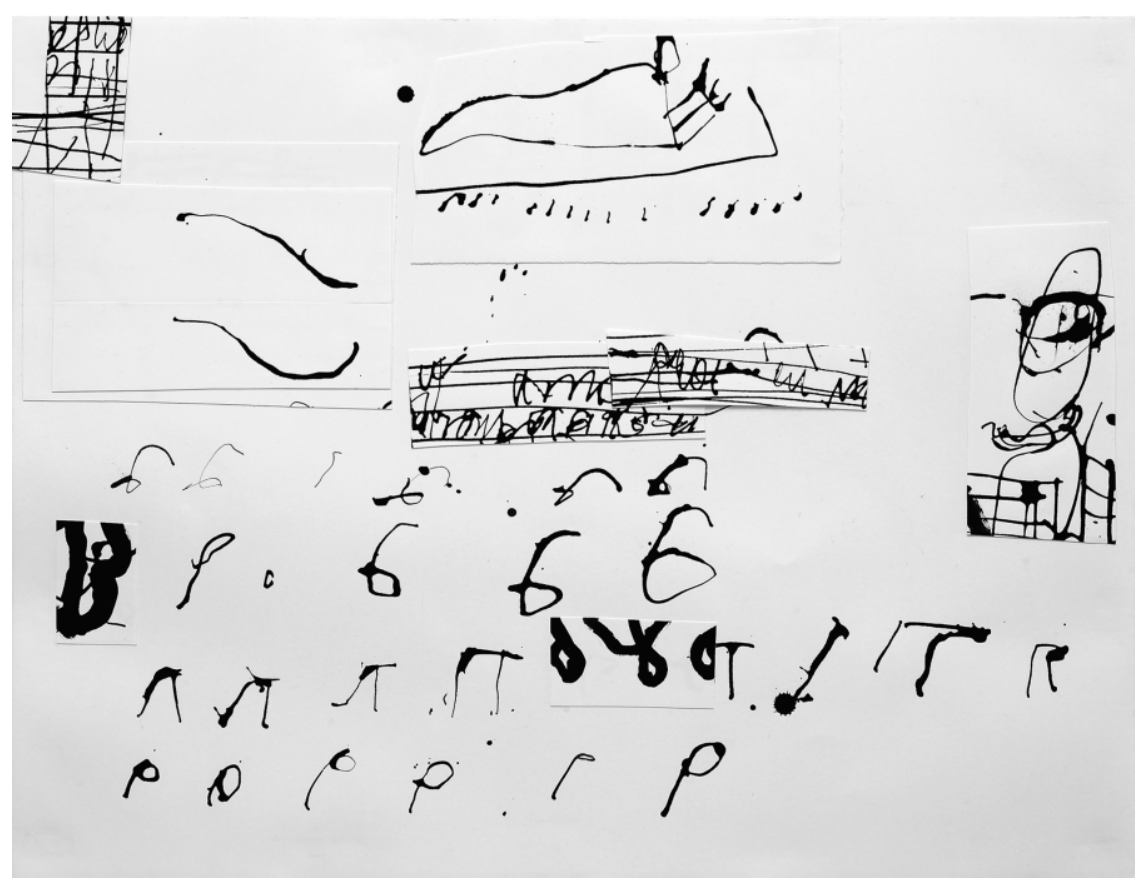

Sans titre $n^{\circ} 2,2007$. Encre et collage sur papier, $52 \times 68 \mathrm{~cm}$.

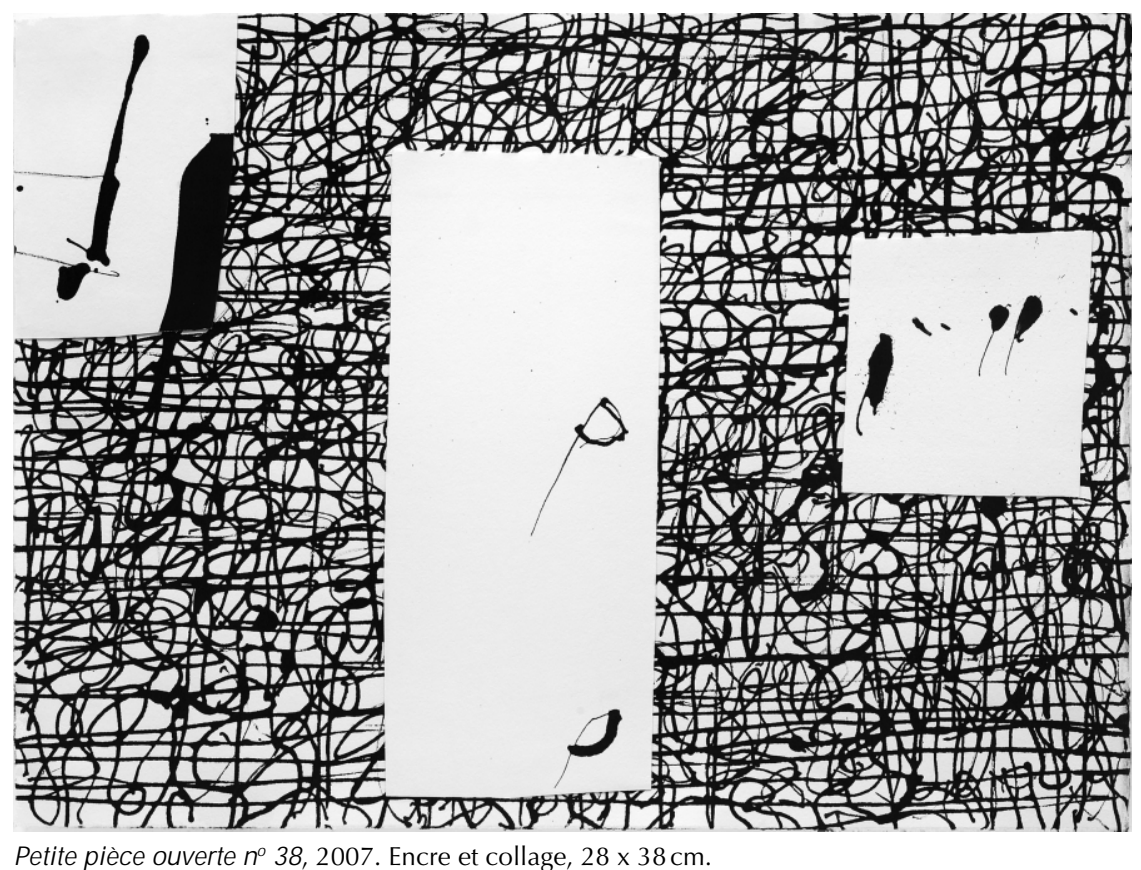




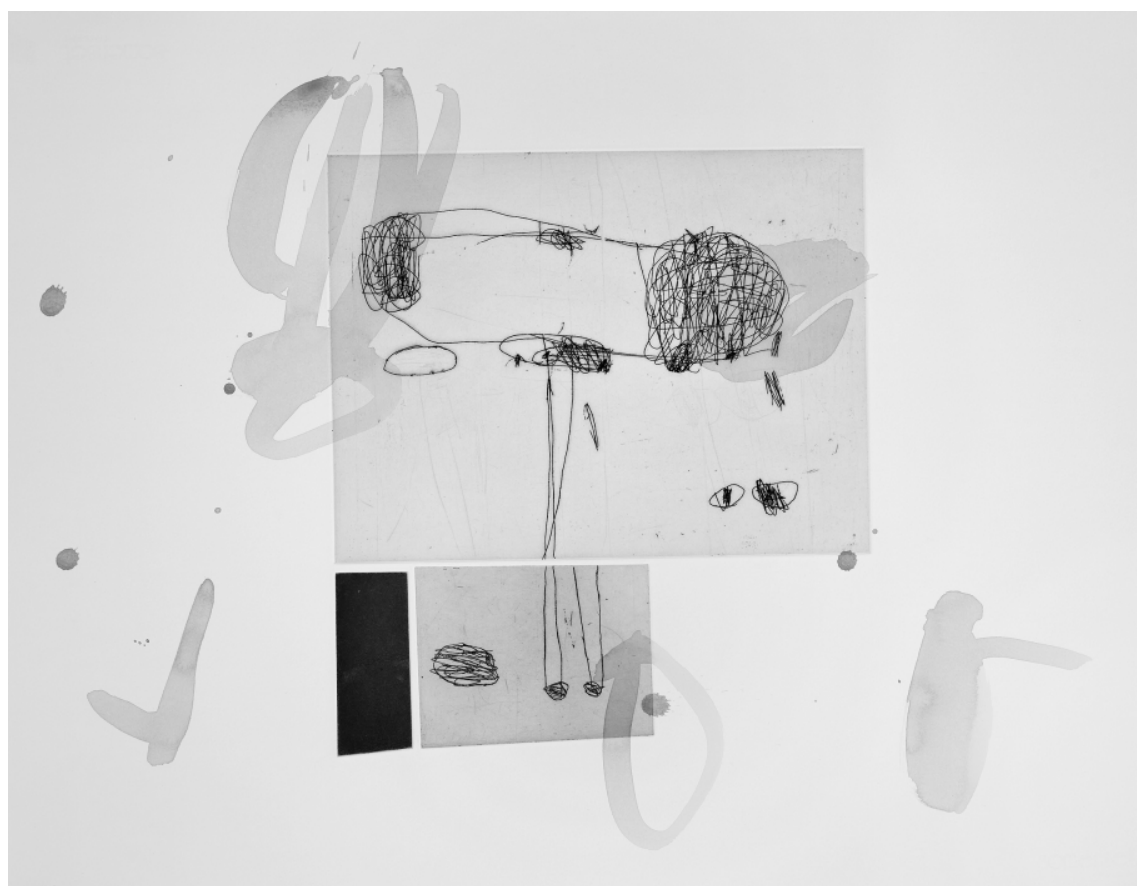

Air de flûte, 2006. Lavis, eau-forte et relief, $56 \times 76 \mathrm{~cm}$. 


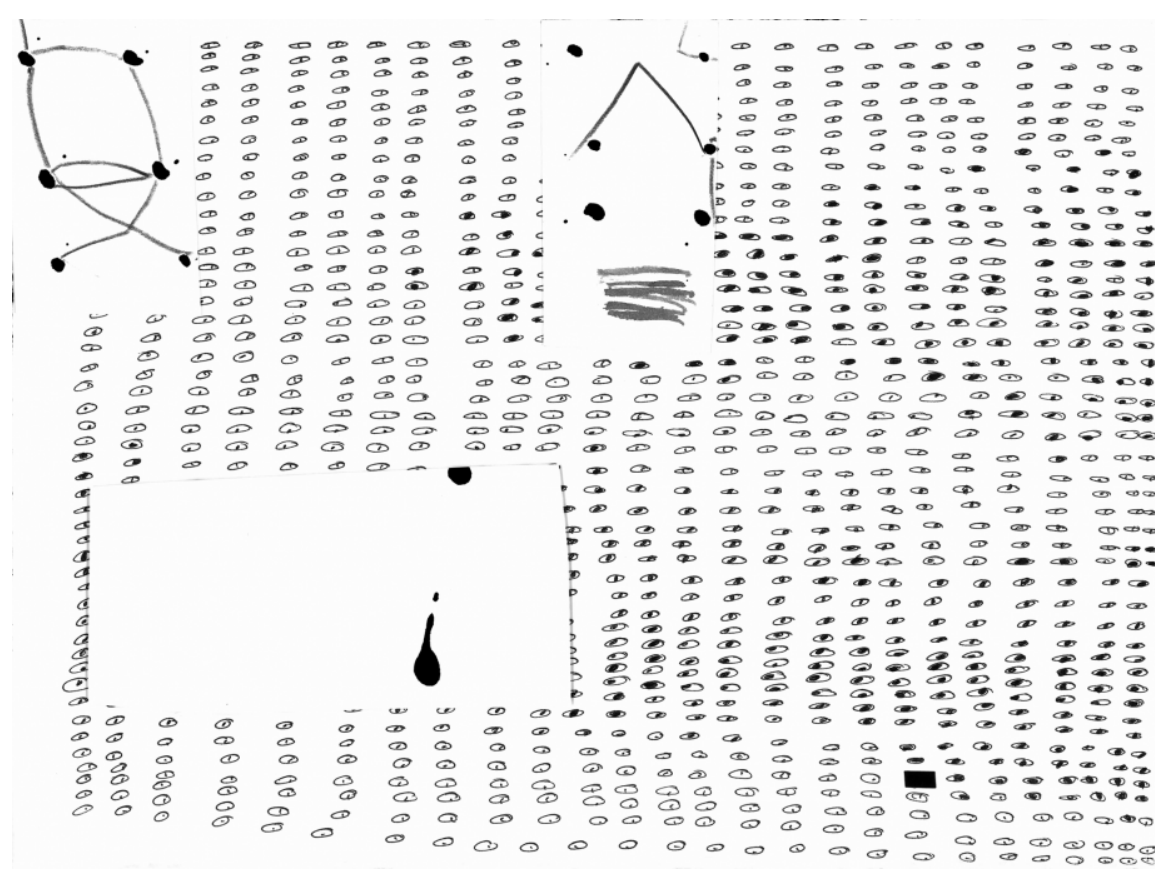

Petite pièce ouverte $n^{\circ} 21,2007$. Encre et collage sur papier, $56 \times 76 \mathrm{~cm}$.

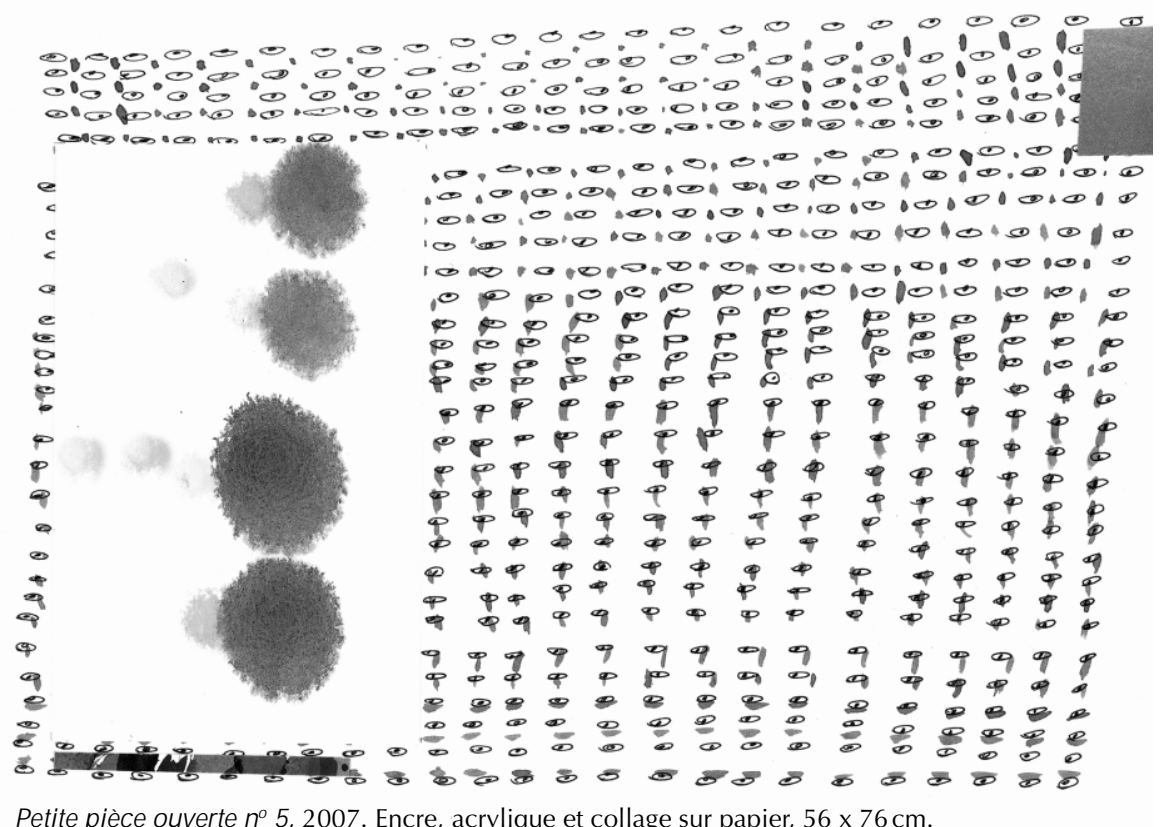



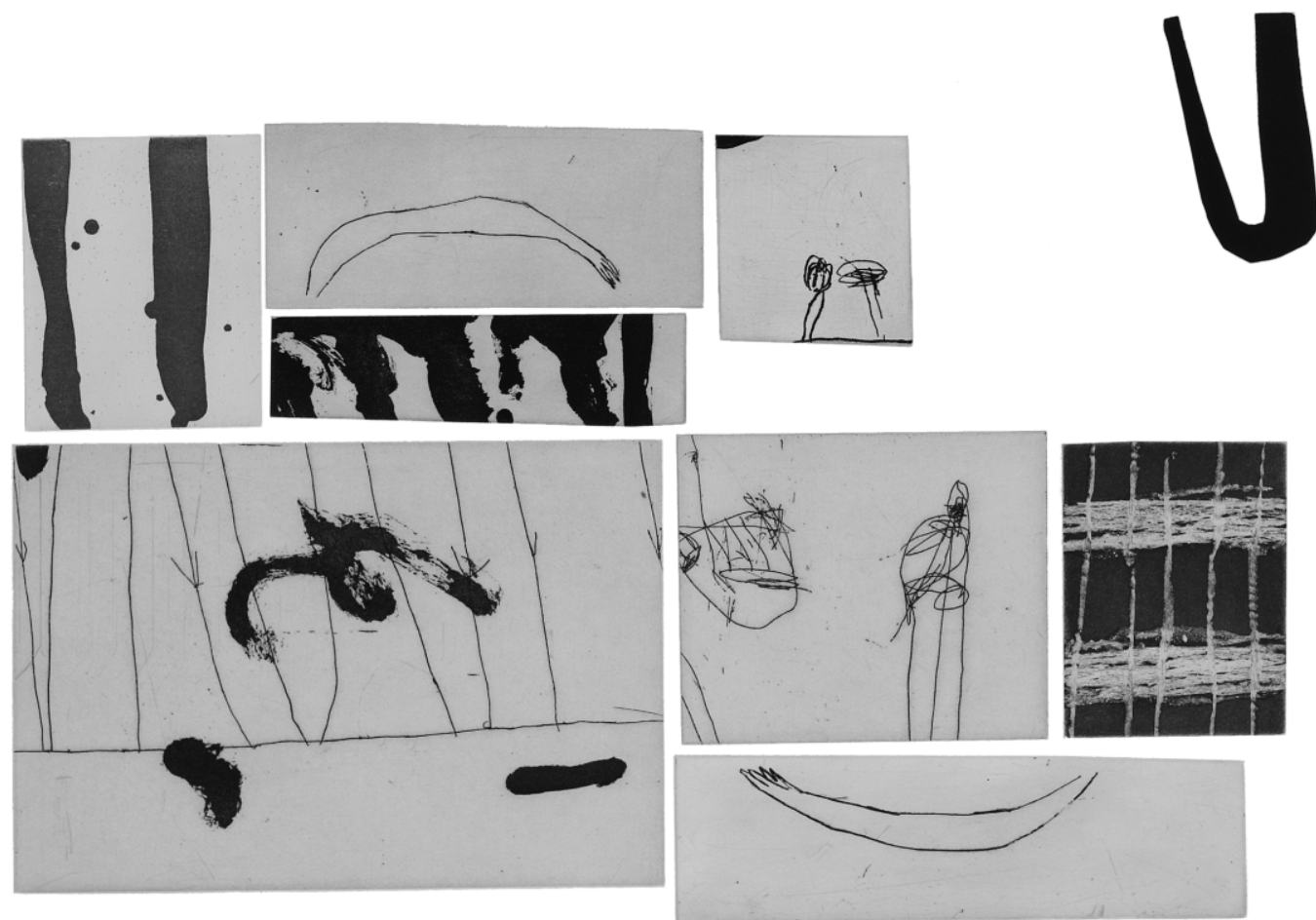

Morceau en U, 2006. Eau-forte et relief, $56 \times 76 \mathrm{~cm}$. 

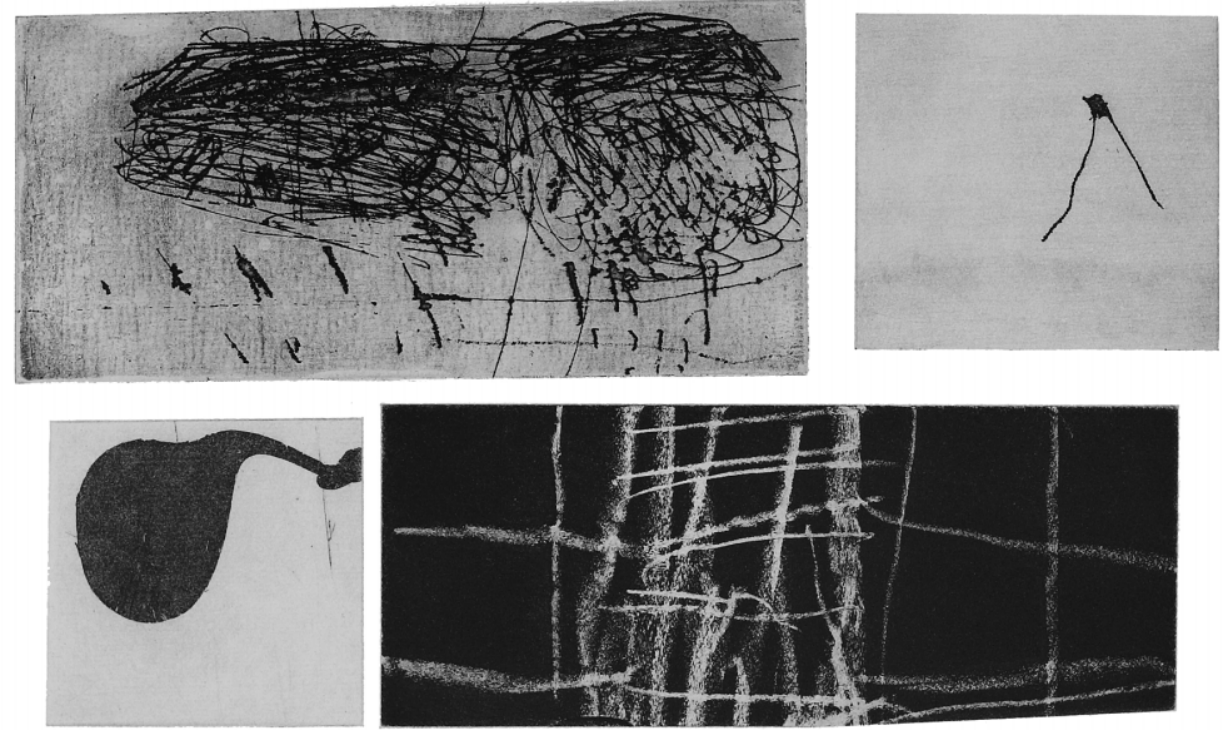

Variation pulsative n², 2006. Eau-forte, $33 \times 57 \mathrm{~cm}$. 